\title{
Design and Characterization of Microstrip Patch Antenna Using Octagonal EBG Periodic Structures
}

\author{
${ }^{1}$ B. Venkateshwar Rao, ${ }^{2}$ Sunita Panda $*^{3}$ Jasjit Suri \\ ${ }^{1}$ Research Scholar, GITAM School of Technology, GITAM, Bengaluru, Karnataka, India \\ bvrao.be@gmail.com \\ ${ }^{2}$ Assistant Professor, Dept. of ECE, GITAM School of Technology, GITAM, Bengaluru, Karnataka, India \\ sunita.nano@gmail.com \\ ${ }^{3}$ Global Biomedical Technologies, Inc., CA, USA \\ jasjit.suri@atheropoint.com
}

Received: 20 ${ }^{\text {th }}$ October 2021, Accepted: $17^{\text {th }}$ November 2021, Published: $31^{\text {st }}$ December 2021

\begin{abstract}
This article divulges regarding the design and characterization of an antenna together with electromagnetic periodic bandgap structures for C-band applications. These periodic structures include the materials to setup different innovative structures that usually finds in many applications for industry and military purpose. Normally substrate with metallic patches are associated along these structures that acquires dimensions usually less than that of its operating wave length $(\lambda)$ when compared to normal design of micro strip antenna found enhancement in efficiency and bandwidth for the structure approximated upon placing it on modified ground surface. For a certain encountered external conditions basically there is less deliberation on optimized design and also on its performance, but maximum literatures concentrate specially on computational aspects of EBG (Electromagnetic Band Gap) structures. The basic design encountered in the paper is associated with a set of octagonal slots within the substrate confined with both ground plane and patch. The dimensions of parameters for designing the antenna model is obtained by the concept of optimization using HFSS (High Frequency Structure Simulator) tool that can resonate at desired frequency. A better return loss of $-21.09 \mathrm{~dB}$ is recorded with this structure when compared with normal conventional patch antenna. The proposed design is resonating at $6.9 \mathrm{GHZ}$.
\end{abstract}

Keywords: EBG structure, octagonal slots, optimization, patch antenna, C-Band frequency range

\section{Introduction}

Generally, antennas are preferred to carry out the information for longer distances i.e. from source to destination, which are playing an important role in case of wireless communication. These antennas will take the information source originated of speech from individual else numerical data from systems.

The basic design of conventional micro strip patch (MSP) antenna's are constructed by using a conducting patch of standard shape when placed on substrate with desired dielectric material associated on a uniform ground plane. The antenna shapes considered may be of square, triangular, rectangular, elliptical, circular etc basically designed using copper material[1,2]. These conventional antennas are preferred and started using gradually due to its familiar properties of having less expensive, low profile and can be directly printed[3].

To enhance the basic parameters of conventional micro strip patch antennas periodic EBG structures are introduced which are considered to be lucrative in wireless communication[4,5]. Most of the investigators also prefer antenna design with various slotted shapes enhancing the parameters at respective frequencies. It is observed that there are also some other advantages of patch antenna like compactness and easy to fabricate. Due to these eminent features it has attracted a lot of analysts to improve the parameters in achieving high gain, wide bandwidth, directiveness etc. 
A basic drawback found in this antenna design structures radiating electromagnetic energy in a particular medium that, some portion of radiations is reflected back along the corner edges which are impotent to radiate defined to be surface waves. In order to overcome these limitations in conventional patch antennas periodic EBG structures are introduced that are identical so as magnetic surface results in a very high impedance surface (HIS).

By properly aligning these EBG periodic structures and taking appropriate design parameter values obtained by optimization concept, it can be managed to suppress surface waves that are radiating at desired frequency levels. Such periodic structures are also regarded as frequency selectors[6,7]. Due to these arrangements mutual coupling between the radiators is also diminished accordingly. Periodic EBG structures are classified in three different types based on their geometrical configurations like one dimensional (used in filtering applications), two dimensional (mushroom type square patches), multy or three dimensional (laying metallic patches both in vertical and also in horizontal directions).

The proposed antenna structure designed is exposed in section II. Periodical EBG structure is enumerated in section III. Antenna analysis together with the help of simulation results are contemplated in section IV. Finally section V is given with conclusion.

\section{Methodology}

\section{Proposed Antenna Design Structure}

The proposed antenna structure designed in this paper is desired to resonate at frequency of $6.9 \mathrm{GHz}$ which comes within the range of C-band[8]. The substrate material considered of FR4 with a loss tangent of 0.02 is designed with octagonal slots[9,10]. Due to the association of enormous parameters that are likely to be continuous, discrete or both in few cases, design flow of antenna process may decelerate and also become complicated. Optimization concept is used in evaluating and fixing scattering, radiation issues related to design parameters for the antenna proposed[11,12]. In recent days the concept of optimization is playing an important role in maintaining the parameter values. The proposed antenna structure is designed using EBG periodic structures with octagonal slots together introducing optimization technique which is suitable for the applications in C-band frequency range with High Frequency Structure Simulator (HFSS) [13].

Optimization technique is an important method of investigating a most acceptable structure used to design when compared with numerous available possibilities[14]. This technique is applied for modelling the design at various steps i.e. to find geometry (like size, structure, orientation etc) material option (having low loss, complex in nature etc) boundaries and together with solution setup. Once, after getting an optimized design structure, it is investigated further to resonate at desired frequency. The parameter values obtained using optimization concept to construct proposed antenna design is given in table 1.

Table 1: Proposed antenna dimensions

\begin{tabular}{|l|l|l|}
\hline Design Parameters & Label & Value (mm) \\
\hline Width of substrate & $\mathrm{W}_{\mathrm{s}}$ & 21 \\
\hline Length of substrate & $\mathrm{L}_{\mathrm{s}}$ & 22 \\
\hline Height of substrate & $\mathrm{H}_{\mathrm{s}}$ & 1 \\
\hline Width of patch & $\mathrm{W}_{\mathrm{p}}$ & 13 \\
\hline Length of patch & $\mathrm{L}_{\mathrm{p}}$ & 10.7 \\
\hline Width of feed & $\mathrm{W}_{\mathrm{f}}$ & 0.9 \\
\hline Length of feed & $\mathrm{L}_{\mathrm{f}}$ & 7 \\
\hline
\end{tabular}


As specified the simplified structure of proposed antenna model which includes ground plane, substrate and patch is depicted in fig 1.

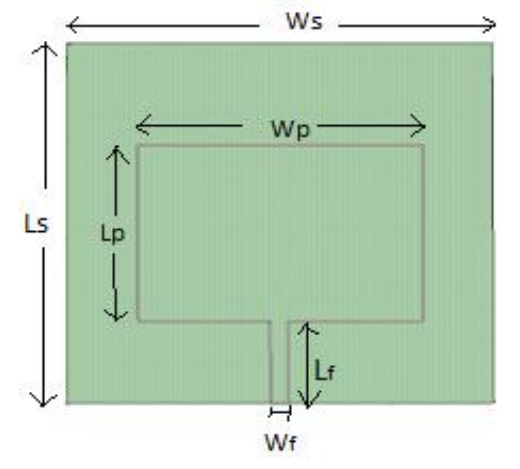

Fig 1: Analytical representation of proposed antenna structure

A group of four EBG arrays arranged at a gap of $1 \mathrm{~mm}$ in its width and a gap of $2 \mathrm{~mm}$ in its length is represented in fig 2. Each element in an array has a rectangular shaped cell of dimensions $10 \mathrm{~mm} \times 10 \mathrm{~mm}$ is accommodated with octagonal slot. Radii of $2 \mathrm{~mm}$ for inner and $3 \mathrm{~mm}$ for outer octagonal slots are considered. The gap between the two complementary rings is maintained of $0.5 \mathrm{~mm}$ and thickness of $0.7 \mathrm{~mm}$ is maintained related to these two octagonal [15]. The total structure of proposed design consisting of rectangular patch antenna with an array of EBG structured octagonal slots are shown in fig 3.

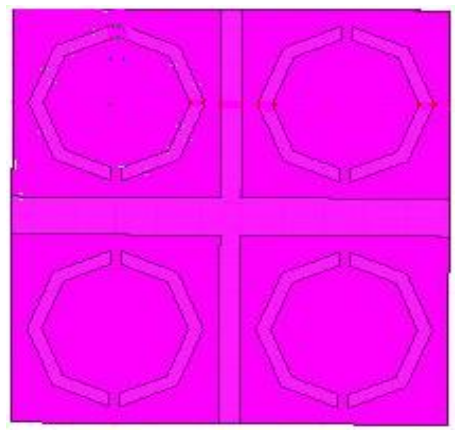

Fig 2: Geometrical structure of EBG Array

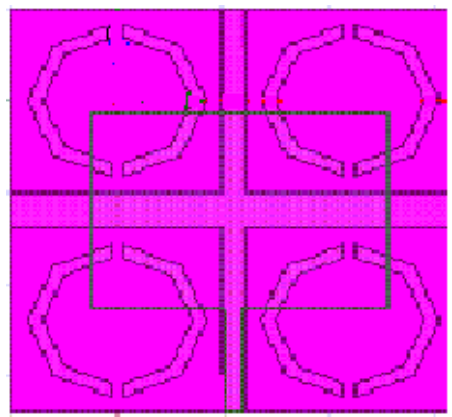

Fig 3: Schematic representation of proposed EBG antenna array 


\section{Results and Discussion}

\section{Analysis of antenna and Experiment Results}

In this section, results obtained from simulation of antenna proposed are short listed. Electromagnetic energy is known to be transferred from source to destination to impart wireless communication. Two important metrics as return loss and VSWR related to the performance of antenna is discussed. Return loss metric can be illustrated taking the ratio of RF waves received to one transmitted by the antenna. Applying the concept of optimization technique for various lengths of patch, the return loss values are recorded as shown in fig 4(a). From the values recorded it is clearly predicted that the return losses for length $12 \mathrm{~mm}$ is about $-26.33 \mathrm{~dB}$, for a length $13 \mathrm{~mm}$ is about $-21.09 \mathrm{~dB}$ and for length $14 \mathrm{~mm}$ is about $-16.57 \mathrm{~dB}$. From the values obtained a good return loss of $-21.09 \mathrm{~dB}$ for a patch length of $13 \mathrm{~mm}$ is well suited for the proposed antenna to resonate closely at desired frequency i.e. 6.93 $\mathrm{GHz}$.

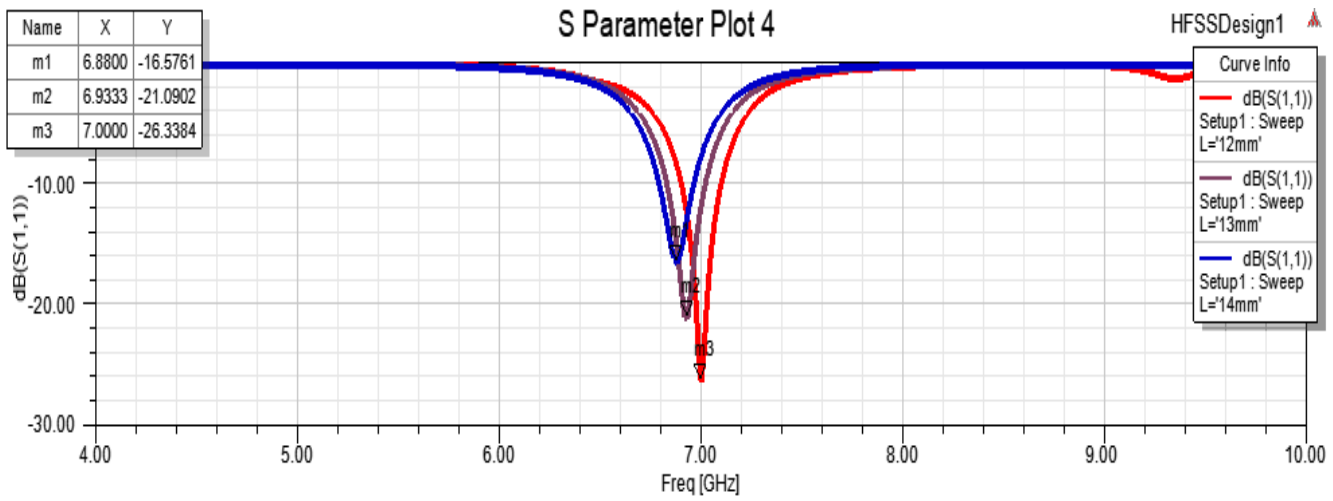

Fig 4(a): Return loss plot

Similarly Voltage Standing Wave Ratio is next metric considered to be an optional approach related to return losses. It is normally defined as the proportion of forward waves to the reflected waves that are emerged proportionately within the range of transmission medium. VSWR value of 1.42 is recorded from the simulation results shown in fig 4(b). The measurement of radiation pattern for the mentioned gain and directivity is depicted in fig 4 (c-d). From the figure shown the values for gain and directivity is recorded as $4.94 \mathrm{~dB}$ and 6.8dB. Fig 4(e-f) depicts the 3D (three dimensional) radiation pattern measurement. 


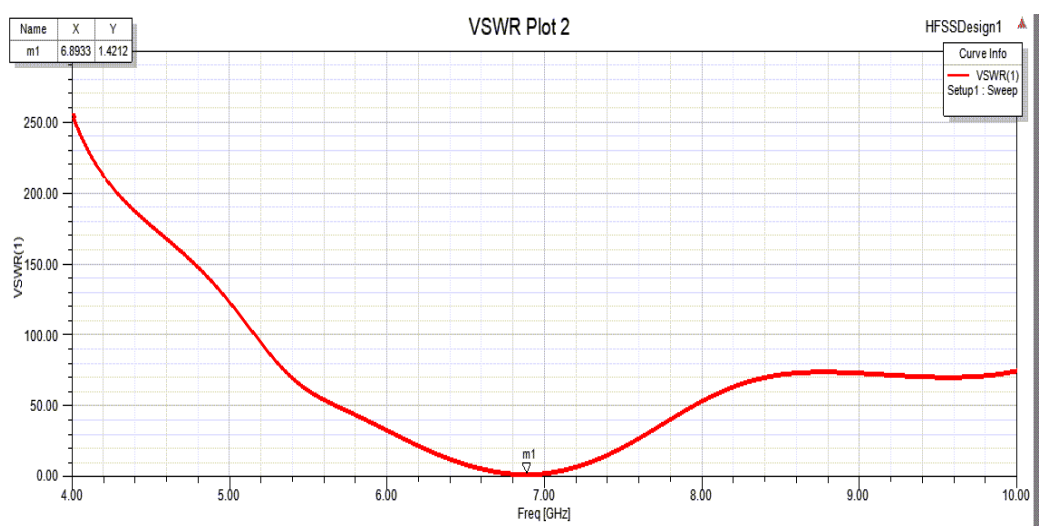

Fig 4(b): VSWR plot

\begin{tabular}{|c|c|c|c|}
\hline Name & Theta & Ang & Mag \\
\hline $\mathrm{m} 1$ & 0.0000 & 0.0000 & 4.9445 \\
\hline
\end{tabular}

\begin{tabular}{|c|c|c|c|}
\hline Name & Theta & Ang & Mag \\
\hline $\mathrm{m} 1$ & 0.0000 & 0.0000 & 6.8056 \\
\hline
\end{tabular}

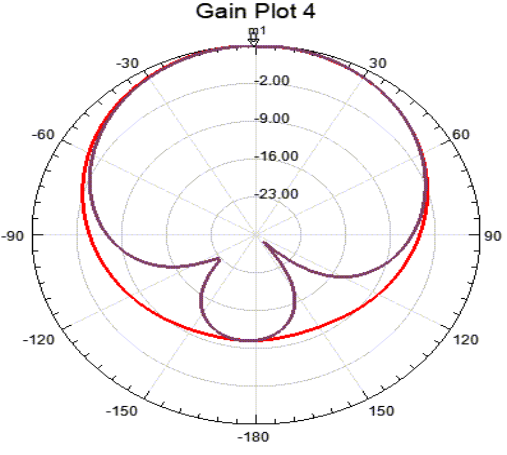

Fig 4(c): Gain plot
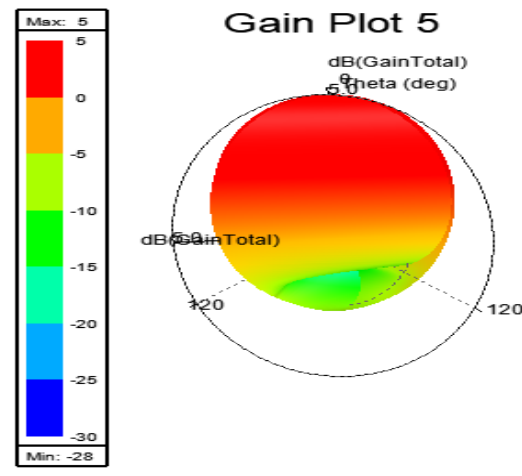

Fig 4(e): 3D-gain plot

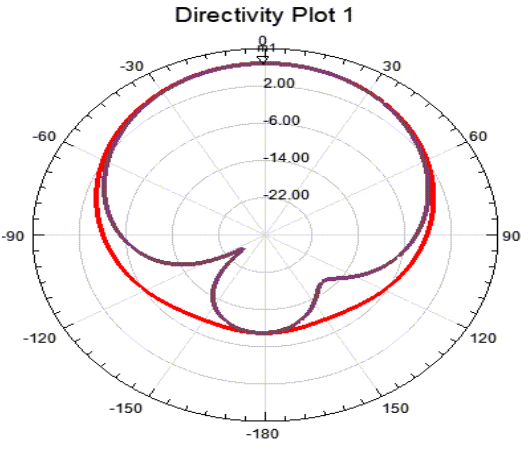

Fig 4(d): Directivity plot

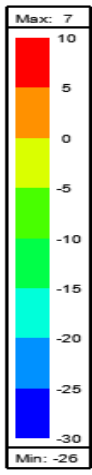

\section{Directivity Plot 3}

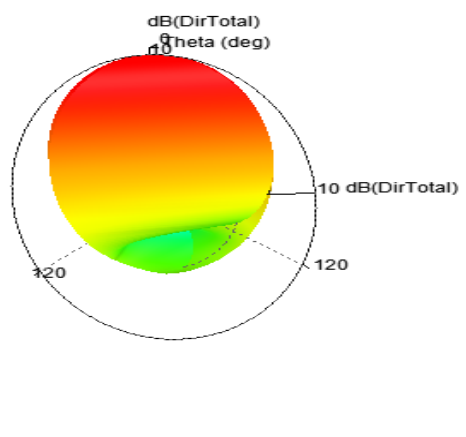

Fig 4(f): 3D-Directivity plot 
The article also discuss the details of polarization effects. Linear polarization is introduced in the paper. When both transmitting antenna and receiving antenna transmits and receives the radiations at phi $=0^{0}$ then these two are said to be co-polarized, nevertheless if receiving antenna receives the radiation at phi $=90^{\circ}$ these two antennas are said to be cross polarized. The co-pole and cross-pole values recorded after simulation is given by 4.8 and -41.45 as shown in fig 5(a). Normally co-pole will have much higher value when compared with cross-pole value that helps in achieving better polarization effects [16].

\begin{tabular}{|c|c|c|c|}
\hline Name & Theta & Ang & Mag \\
\hline $\mathrm{m} 1$ & 0.0000 & 0.0000 & 4.8023 \\
\hline $\mathrm{m} 2$ & 14.0000 & 14.0000 & -41.4551 \\
\hline
\end{tabular}

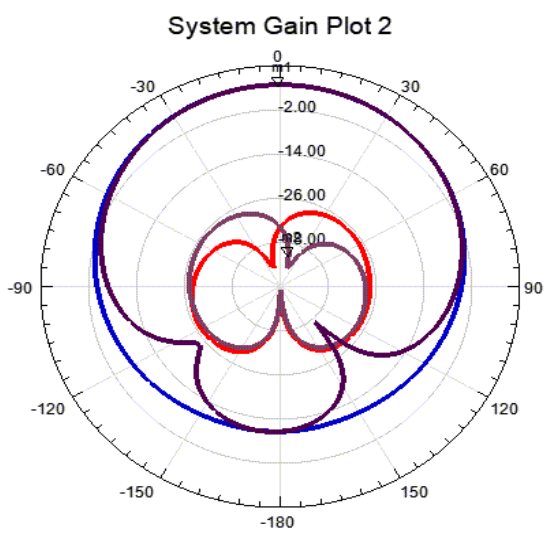

Fig 5(a): Co-pole and Cross-pole plot

Fig 5(b) depicts the movements of current direction in antenna which is observed of having fields (current) carrying maximum at the centre and decelerates as it moves outward. One more antenna parameters is introduced i.e. Half Power Beam Width (HPBW) generally measured in terms of degrees that is defined as angular representation of a radiation pattern decreased in its magnitude by $50 \%$ (or $-3 \mathrm{~dB}$ ) when compared with the peak of its main beam. When the proposed antenna radiating at $6.9 \mathrm{GHz}, \mathrm{HPBW}$ is measured at phi $=0$ and $\mathrm{phi}=90^{\circ}$ as shown in fig $5(\mathrm{c})$. The discussions made so far provides an outstanding study of parametric analysis for antenna that can be designed accordingly. 


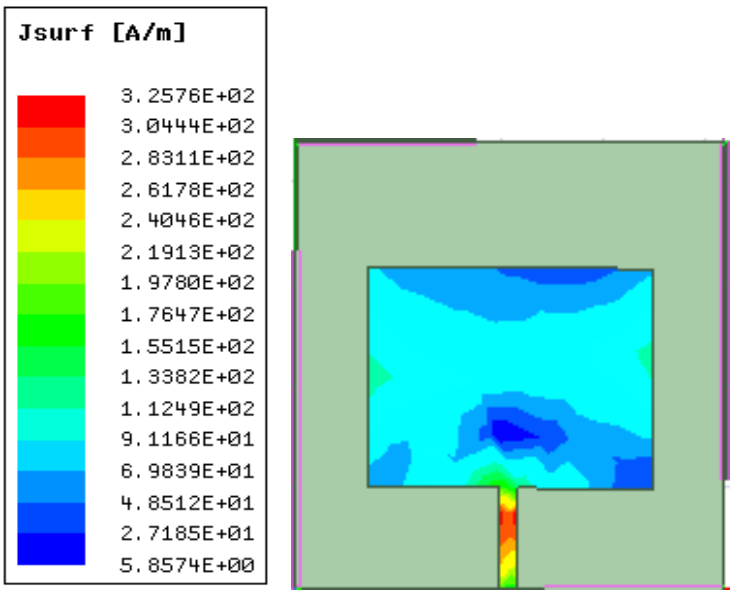

Fig 5(b): Fields (Current) of Antenna plot

\begin{tabular}{|l|c|}
\hline \multicolumn{1}{|c|}{ Curve Info } & xdb10Beamwidth(3) \\
\hline $\begin{array}{l}\text { dB(GainTotal) } \\
\text { Setup1 : LastAdaptive } \\
\text { Freq='6.9GHz' Phi=0deg" }\end{array}$ & 90.7304 \\
\hline $\begin{array}{l}\text { dB(GainTotal) } \\
\text { Setup1: LastAdaptive } \\
\text { Freq='6.9GHz' Phi="90deg" }\end{array}$ & 87.7869 \\
\hline
\end{tabular}

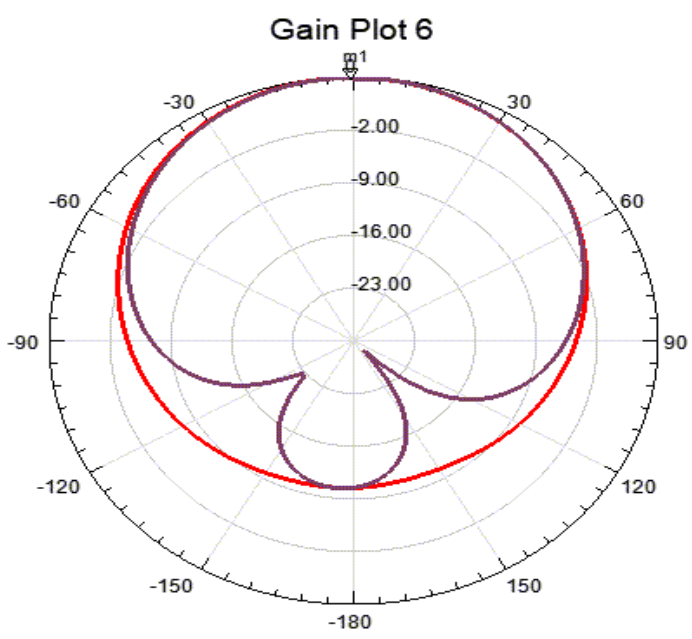

Fig 5(c): Half Power Beam Width (HPBW) plot

\section{Conclusion}

A compact periodic EBG antenna array provided with octagonal slots is detailed in this article using the concept of optimization technique that best suits for C-Band applications simulated using HFSS. Smart designing of antennas can be achieved by using the concept optimization technique effectively. Based on this concept the proposed antenna providing a gain value more than $4.94 \mathrm{~dB}$ and with directivity of $6.8 \mathrm{~dB}$ when compared with conventional micro strip patch antennas when resonating at same desired frequency $6.9 \mathrm{GHz}$. Bandwidth of proposed antenna can still be enhanced by modifying the radii and space between the octagonal split slots. The efficiency ' $\eta$ ' of the proposed radiating antenna is achieved up to $73 \%$ showing applicability of the respective radiator at desired frequency. 


\section{References}

1. Taksala Devapriya Amalraj.,Robinson Savarimuthu (2019). Design and Analysis of Microstrip Patch Antenna Using Periodic EBG Structure for C-Band Applications. Springer Science+ Business Media, LLC, part of Springer Nature 2019.

2. He Huang, Xiaoping Li and Yanming Liu.,(2019) A Low Profile, Single-ended and Dual-polarized Patch Antenna for 5G Application., IEEE Transactions on Antennas and Propagation.

3. K. Praveen Kumar et.al, B. Amulya and B.Venkateshwar Rao, Compact Multiband Printed Antenna Design and Analysis., International Journal of Control and Automation Vol. 13, No. 3, (2020), ISSN: 2005-4297, pp. 58-63.

4. K.Praveen Kumar et.al, "Optimization of EBG structure for mutual coupling reduction in antenna arrays; a comparitive study" IJET, Vol-7, No-3.6, Special issue-06, 2018.

5. K.Praveen Kumar et.al, "Active PS EBG structure design for low profile steerable antenna applications" JARDCS, Vol-10, Special issue-03, 2018.

6. Chatterjee, A., \& Parui, S. K. (2017). Frequency-dependent directive radiation of monopole-dielectric resonator antenna using a conformal frequency selective surface. IEEE Transactions on Antennas and Propagation, 10(1109), $1-7$.

7. K.Praveen Kumar et.al, " Surface wave suppression band, In phase reflection band and High Impedance region Of 3D EBG Characterization" IJAER, Vol 10, No 11, 2015

8. K.Praveen Kumar et.al, "Fractal Array antenna Design for C-Band Applications", IJITEE, Volume-8 Issue-8 June, 2019.

9. B. Premalatha, M. V. S. Prasad and M. B. R. Murthy, Compact Hexagonal Monopole Antenna, Indian Journal of Science and Technology, Vol 10(19), May 2017 ISSN (Print) : 0974-6846 ISSN (Online) : 0974-5645.

10. B.Venkateshwar Rao et.al, Praveen Kumar Kancherla, B.Amulya, Multiband slotted Elliptical printed Antenna Design and Analysis JMCMS, Vol.-14, No.-4, July-August (2019) pp 378-386.

11. K.Praveen Kumar et.al, "Design and characterization of Optimized stacked electromagnetic band gap ground plane for low profile patch antennas" IJPAM, Vol 118, No. 20, 2018, 4765-4776.

12. M. Pokorn and J. Horak, "Design and global multi-objective optimization of planar tri-band antenna," in Proc. 17th Int. Conf. Radioelektronika, pp. 1-5, April 2007.

13. Siyang SUN, Yinghua LV, Jinling ZHANG, Zhidong ZHAO and Fangming RUAN (2010). Optimization Based on Genetic Algorithm and HFSS and Its Application to the Semiautomatic Design of Antenna, 978-1-4244-57083/10/\$26.00@2010 IEEE.

14. K.Praveen Kumar et.al. "Optimization of EBG structure for mutual coupling reduction in antenna arrays; a comparitive study" IJET, Vol-7, No-3.6, Special issue-06, 2018. page 13- 20.

15. B. Premalatha, M. V. S. Prasad b and M. B. R. Murthy, (2018) Compact Penta Band Notched Antenna Using Concentric Rings with Splitter Bricks for Ultra Wide Band Applications, Journal of Communications Technology and Electronics, ISSN 1064-2269, Vol. 63, No. 12, pp. 1379-1385.

16. K.Praveen Kumar, "Circularly Polarization of Edge-Fed Square Patch Antenna using Truncated Technique for WLAN Applications", IJITEE, Volume-8 Issue-8 June, 2019. 